\title{
EFFECT OF SUPPLY CHAIN STRUCTURE AND POWER DYNAMICS ON R\&D AND MARKET PERFORMANCES
}

\author{
Seung Ho YOO${ }^{1}$, Yong Won SEO ${ }^{2}$ \\ ${ }^{1}$ SunMoon University, Asan, 336-708 Republic of Korea \\ ${ }^{2}$ Chung-Ang University, Seoul, 156-756 Republic of Korea \\ E-mails: ${ }^{1}$ shy1228@sunmoon.ac.kr; ${ }^{2}$ seoyw@cau.ac.kr (corresponding author)
}

Received 08 November 2016; accepted 03 May 2017

\begin{abstract}
This study is the first investigating the effect of supply chain structure and players' power dynamics on a supply chain's R\&D and market performances. We consider a three-stage supply chain, consisting of a R\&D firm, a manufacturer, and a seller. We develop six supply chain models based on various structures and power dynamics, and reveal important implications by comparing the models. R\&D performance is a function of supply chain structure; an integrated supply chain can expect improved performance. The selling price is a function of power dynamics, and is higher when R\&D has bargaining power. Market and profit performances are higher in an integrated, manufacturer-led supply chain. The manufacturer's role must be revisited; due to its location in the middle of the supply chain, the manufacturer can directly control the overall chain. On the other hand, a R\&D firm could innately be associated with marginalization issues. Therefore, it is important for the R\&D firm to have a systematic understanding of the entire system. This study contributes to the literature and practice by not only bridging the gap in the previous studies but also offering important managerial implications.
\end{abstract}

Keywords: R\&D, supply chain structure, power dynamics, product quality, pricing, principal-agent paradigm.

JEL Classification: C71.

\section{Introduction}

In a contemporary business environment, many firms enjoy advantages in quality, cost, and/or technological expertise. They maximize return on resources by focusing on core competencies and strategically outsourcing non-core activities. Therefore, a common practice is supply chain-based business management, with functions that are decentralized to specialized partners. The research and development (R\&D) function is no exception.

More complicated product technologies require a firm to be more reliant on its partners' complementary capabilities. Controlling all required technologies is inefficient, and of-

This article has been corrected since first published. Please see the statement of correct (http://doi.otg/10.3846/16111699.2017.1356445). 
ten virtually impossible. Therefore, the R\&D function is often decentralized across the supply chain. R\&D outsourcing and specialization is increasing, with a significant proven contribution to firms' performance (Han, Bae 2014). For example, Apple Inc. focuses on R\&D and marketing, while delegating production to key suppliers in Asian countries. While Samsung Electronics is responsible for both R\&D and manufacturing functions, it often relies on its external partner's R\&D capability. It applied Qualcomm's AP (application processor) technology to its Galaxy S5 smartphone, paying patent royalties at $2.5-5.0 \%$ of the retail price (PhoneArena 2015). Strategic R\&D specialization and outsourcing is not limited to the manufacturing industry. For example, Marvel Studios, famous for superhero movies, concentrates on developing fictional contents and moviemaking, while decentralizing the films' distribution to Walt Disney Studios. The key role of Tencent, a Chinese IT service provider, is the distribution of a number of online games through its portal; however, the majority of games are developed by R\&D partners in many countries.

The above examples demonstrate that a supply chain's structure varies. There is neither a single fixed structure nor a fixed power relationship to gain a successful R\&D result and its distribution. Moreover, a supply chain may need to change its form to improve. In Samsung's case, it recently eliminated its dependency on Qualcomm and internalized the R\&D of AP by adopting an in-house AP for its Galaxy S6 model (PhoneArena 2015). Firms must constantly determine which functions to outsource and which functions to focus on; it is important to understand the characteristics of possible supply chain structures, and also to find the best structure for superior performance. Therefore, this study introduces various supply chain structures, considering possible combinations of processes as well as power dynamics between players. It investigates the differentiation of R\&D and market performances through supply chain structure-related decisions.

A three-stage supply chain is considered, comprising a specialized R\&D company, a manufacturer, and a seller. The R\&D company can be a critical supplier, a patent-holding company, or a fashion designer, whose result has a critical impact on product quality as well as on overall supply chain performance. For simplicity, our discussion is limited to cases in which either the R\&D company or the manufacturer has bargaining power over other players (e.g., in the high-tech electronics industry). By considering possible combinations of processes and power dynamics, six different supply chain models, based on the principal-agent paradigm, are introduced. Performances are compared, and distinct characteristics of the six supply chain models are revealed. Specifically, the decisions in each model are characterized by (1) the contracts: transfer payments for the R\&D result and item supply; (2) the R\&D performance: the resulting quality level; (3) the selling price; (4) the market performance: demand requests; and (5) the profit performances of $R \& D$, the manufacturer, the seller, and the entire supply chain. Despite its growing practical importance, the choice of supply chain structures and its effect on R\&D performance has not yet been extensively studied. We contribute to this body of knowledge by identifying the characteristics of various supply chain structures, and by providing important implications. 


\section{Literature review}

Literature relevant to this study is: (1) strategic R\&D outsourcing and specialization, and (2) supply chain structural design.

A number of analytical studies have dealt with R\&D performance in a decentralized system. Many studies deal with a non-cooperative game, considering competition between a patent-holding R\&D firm and the firms that license the patent technology (Kamien et al. 1992; Wang 2002; Sen 2005; Kishimoto et al. 2011; Kishimoto, Watanabe 2017). These studies focus on the type of license contract that can yield superior performance for an outside R\&D firm, in most cases comparing fixed fee and per unit royalty contracts. However, most R\&D studies involve cost-reducing technology. Studies more relevant to ours, such as Stamatopoulos and Tauman (2008), Li and Wang (2010) and Tian (2016), address quality innovation through R\&D and its effect on market performance. They reveal that per unit royalty licensing guarantees a superior result for $R \& D$ firms. However, these studies are also based on a non-cooperative game, focusing on competition between players. They differ from this study, which focuses on a cooperative game, based on the principal-agent paradigm framework in a three-stage supply chain in which an R\&D firm, a manufacturer, and a seller collaborate. The collaboration that yields superior R\&D, market, and profit performances, according to different supply chain structures and power dynamics, is revealed.

Decentralization of certain functions inevitably results in structural supply chain changes, which often accompanies double or multiple marginalization issues among players. It points to the phenomenon that each firm in a supply chain (e.g., a manufacturer and a retailer), sets its price above its marginal cost. This would happen multiple times if there are a number of firms at different vertical levels, subsequently leading to inefficiency and a loss of welfare across the entire supply chain (Spengler 1950). Two primary approaches have been used to mitigate this negative aspect of decentralization. One is supply chain contract design, to control and/or coordinate the decisions of overall supply chain players. Diverse literature addresses this issue, such as the works of Cachon and Lariviere (2005), Ding and Chen (2008), and Zhao et al. (2010).

A more relevant approach to this study involves directly addressing decisions regarding supply chain structure. Corbett and Karmarkar (2001), Carr and Karmarkar (2005) study optimal decisions on order quantity and price in a multi-echelon supply chain. Their models consider within-tier competition, but not between-tier contracts; hence, they do not incorporate multiple marginalization issues among players. Studies have also examined the structural design of a closed-loop supply chain. For example, Savaskan and van Wassenhove (2006) examine joint decisions on selling price, and the return rate of a used item. They consider various reverse channel structures for the used item collection by incorporating various collectors under incentive contracts. Shi and Min (2013) also investigate a closed-loop supply chain's structural design problem. These studies primarily focus on the path difference of reverse channels involving different participants. In contrast, this study considers vertical integration and decentralization of supply chain processes, including R\&D, manufacturing, and sales, as well as different 
power dynamics among the three players, and identifies the most efficient structure. The work of Majumder and Srinivasan (2008) is closely related; it models a multi-tier supply chain network and investigates contract issues involving double marginalization. Similar to this study, they also investigate the effect of changes in power dynamics among players. However, they model the supply structure as given and static; we investigate various supply chain structure options by considering process separation and integration. The study of Lu et al. (2014) is similar to this one, investigating the choice of outsourcing or self-management as supply chain governance. It reveals that the optimal governance model depends on supply chain characteristics such as the costs of knowledge transfer and compliance. However, it differs from this work in that it does not consider different types of power dynamics and R\&D concerns, while focusing on whether or not to outsource a general supply chain process to a third party. In summary, while there have been previous studies dealing with structural supply chain design issues, one that directly addresses $R \& D$ decentralization and its effect on the entire supply chain - as in the present study - is difficult to find.

\section{Basic model formulation}

This study considers consumer products, such as electronic appliances or vehicles. Consumers' buying intention is primarily affected by product quality and price. Therefore, consumers' demand $D$ is defined as follows:

$$
D=\alpha-\beta p+\gamma q,
$$

where $\alpha$ is the demand potential, $p$ and $q$ are the selling price and quality of the product, respectively, and $\beta$ and $\gamma$ are the respective coefficients.

The selling price $p$ negatively affects $D$ in (1) as in many previous studies, such as Raz and Ovchinnikov (2015), Yoo et al. (2015) and Yoo and Kim (2016). Product quality q, which represents $\mathrm{R} \& \mathrm{D}$ performance, enhances $D . q$ is regarded as a single composite measure, as in Karmarkar and Pitbladdo (1997), Banker et al. (1998) and Wang and Shin (2015). Therefore, the value of $q$ is regarded as a sum of measurable quality attributes, such as performance, feature, and reliability. This approach is commonly observed in quality management research, such as Shi et al. (2001), who define quality as the weighted sum of multiple attributes.

We consider three supply chain processes: research and development (R\&D), manufacturing, and sales, which jointly determine product quality and selling price for profit maximization.

The R\&D process invests in technology to enhance the quality of the product $q$. The R\&D output is delivered to the manufacturing process in the form of new product technology, knowledge, engineering skills, and others, for which the manufacturing process pays the $\mathrm{R} \& \mathrm{D}$ process. Therefore, the profit $\Pi_{r}$ of the $\mathrm{R} \& \mathrm{D}$ process is defined as follows:

$$
\Pi_{r}=T_{m r}-\lambda q^{2}
$$

where $\lambda q^{2}$ is the $\mathrm{R} \& \mathrm{D}$ expenditure to achieve the quality $q$. The coefficient $\lambda$ represents 
the magnitude of the cost. We define the R\&D cost as increasing and convex in $q$, as in the literature related to quality investment, such as Karmarkar and Pitbladdo (1997), Banker et al. (1998) and Wang and Shin (2015). $T_{m r}$ is the transfer payment from the manufacturing to the R\&D process, as compensation for the transfer of R\&D output. We will define $T_{m r}$ in the next section, differentiated for the power dynamics between players.

The manufacturing process utilizes the R\&D output, and delivers the product to the sales process, which in turn determines the price of the product $p$ and sells it to consumers. Therefore, the profits of the manufacturing and sales processes $\Pi_{m}$ and $\Pi_{s}$ are defined, respectively, as:

$$
\begin{gathered}
\Pi_{m}=T_{s m}-c D-T_{m r}, \text { and } \\
\Pi_{s}=p D-T_{s m},
\end{gathered}
$$

where $c$ is the unit production cost. $T_{s m}$ is the transfer payment from the sales process to the manufacturing process, incurred by the product supply. We utilize the wholesale price contract to describe the interaction, which is most commonly used in practice. Therefore, $T_{s m}$ can be defined as follows, where $w$ is the unit wholesale price:

$$
T_{s m}=w D \text {. }
$$

The supply chain models in the next section involve different combinations of processes and power dynamics among players, characterized by the profit functions in (2-4).

\section{Supply chain models}

A three-stage supply chain is considered, comprising an R\&D company (R\&D, henceforth), a manufacturer, and a seller. The following assumption is necessary to characterize the power dynamics between players:

Assumption 1. The supply chain's focal company is either $R \& D$ or the manufacturer, while their bargaining power is greater than the seller's.

This discussion focuses on a situation in which either R\&D or the manufacturer dominates the seller - a dynamic that is commonly observed in the high-tech industry, where R\&D and the manufacturer play important roles. For example, many manufacturers, such as GM and Toyota, lead supply chains in the automotive industry. Recently, companies focusing on R\&D capability - with unique expertise in technologies and relevant patents - have gained bargaining power over manufacturers and other supply chain players (e.g., Qualcomm, Inc. in the telecommunications industry).

Assumption 2. The player with greater bargaining power acts as a Stackelberg leader, who, in turn, makes decisions regarding the contract terms and processes integration.

Second, it is assumed that all players have access to the same information, as in many studies based on the cooperative Stackelberg game. The Stackelberg leader (i.e., either $\mathrm{R} \& \mathrm{D}$ or the manufacturer) offers contract terms using its foresight about other players' actions, and determines the integration of processes. 
Assumption 3. An existing supply chain is considered.

We focus on comparing the performances of existing supply chains, which are not newly established but currently operating supply chains, where their decisions regarding supply chain structure have already been made.

Six supply chain models with different combinations of processes and power dynamics are investigated, based on these assumptions: (1) Case FI: full integration (FI) of R\&D (R), manufacturing (M), and sales (S) processes; (2) Case RM: integration of R\&D and manufacturing processes; (3) Case MS-R: integration of manufacturing and sales processes, with R\&D as the Stackelberg leader; (4) Case MS-M: integration of manufacturing and sales processes, with the manufacturer as the leader; (5) Case PD-R: pure decentralization (PD), with R\&D as the Stackelberg leader; and (6) Case PD-M: pure decentralization, with the manufacturer as the leader. Figure 1 illustrates the structures and power dynamics of the six supply chain cases:

(a) Case FI

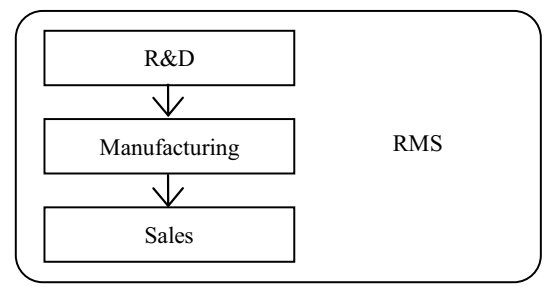

(c) Case MS-R

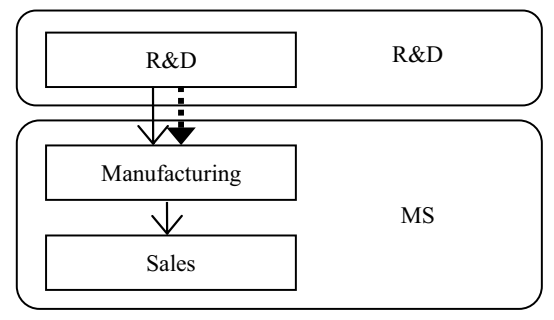

(e) Case PD-R

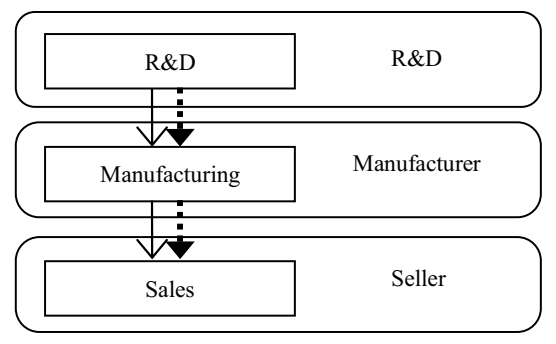

(b) Case RM

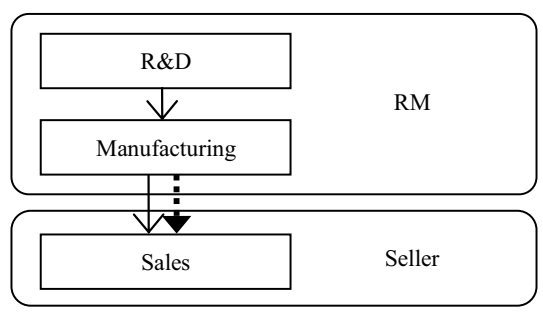

(d) Case MS-M

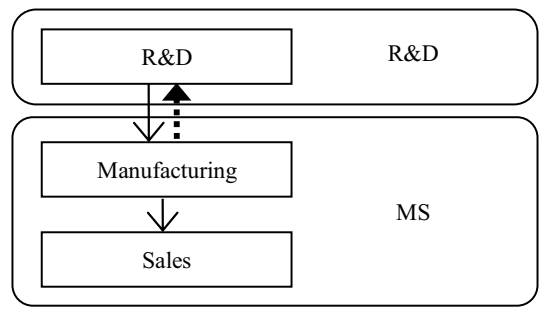

(f) Case PD-M

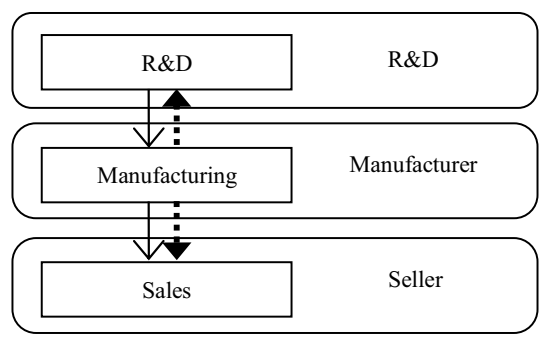

Fig. 1. Supply chain models (R: R\&D, M: manufacturer, S: seller; solid line: inventory or technology flow, dotted line: contract offer) 


\subsection{Case FI: full integration of $R \& D$, manufacturing, and sales processes}

Case FI deals with a fully integrated supply chain, and can be considered an ideal situation with no opportunistic behavior of players. We regard this coordinated Case FI as a benchmark for other cases. The player that integrates overall processes simultaneously plays the roles of R\&D, manufacturer, and seller (RMS), and its profit $\Pi_{R M S} F I$ becomes the same as the profit of the entire supply chain $\Pi^{F I}$, in which the superscript FI denotes Case FI:

$$
\Pi_{R M S}^{F I}=\Pi^{F I}=\Pi_{r}+\Pi_{m}+\Pi_{s}=(p-c) D-\lambda q^{2},
$$

where $D, \Pi_{r}, \Pi_{m}$ and $\Pi_{s}$ are in (1-4), respectively. This fully integrated situation does not need to consider the interaction between players. Therefore, the problem of Case FI can be simply defined as:

$$
\text { Maximize } \Pi_{R M S}^{F I}(p, q) \text {. }
$$

By partially differentiating $\Pi_{R M S}{ }^{F I}(p, q)$ in (7) with respect to $p$ and $q$, we obtain the first-order necessary conditions (FONCs), $\left(\partial \Pi_{R M S} F I / \partial p\right)=\alpha-2 \beta p+\beta c+\gamma q=0$ and $\left(\partial \prod_{R M S} F I / \partial q\right)=\gamma p-\gamma c-2 \lambda q=0$. Then, by simultaneously solving FONCs above, we obtain the optimal $p^{F I^{*}}$ and $q^{F I^{*}}$ as:

$$
\begin{gathered}
p^{F I^{*}}=\frac{2 \lambda(\alpha-\beta c)}{4 \beta \lambda-\gamma^{2}}+c, \text { and } \\
q^{F I^{*}}=\frac{\gamma(\alpha-\beta c)}{4 \beta \lambda-\gamma^{2}} .
\end{gathered}
$$

As observed in (8) and (9), we can expect a higher optimal selling price $p^{F I^{*}}$ and product quality $q^{F I^{*}}$ when there is a larger consumer base (an increase in $\alpha$ ); consumers are less sensitive to the selling price (a decrease in $\beta$ ); or consumers prefer a higher quality product (an increase in $\gamma$ ). When quality enhancement needs more investment (an increase in $\lambda$ ), we cannot expect higher product quality. The decision structures of the optimal selling price and quality are also similar in other supply chain cases (see Table 1).

To ensure $p^{F I^{*}}>0$ and $q^{F I^{*}}>0$ and the concavity of $\Pi_{R M S}{ }^{F I}$, the conditions below are necessary:

$$
\begin{gathered}
\alpha>\beta c, \text { and } \\
4 \beta \lambda-\gamma^{2}>0 .
\end{gathered}
$$

The demand potential $\alpha$ must be sufficiently large in Condition (10) to ensure interior solutions. Condition (11) is from the second-order sufficient condition (SOSC), that is, $\left|\mathrm{H}_{2}\right|=\left(\partial^{2} \Pi_{R M S}^{F I} / \partial p^{2}\right)\left(\partial^{2} \Pi_{R M S}^{F I} / \partial q^{2}\right)-\left(\partial^{2} \Pi_{R M S} F I / \partial p \partial q\right)^{2}=4 \beta \lambda-\gamma^{2}>0$. To ensure the optimal solution by guaranteeing the concavity of the profit function, there must be a certain balance between the positive $(\gamma)$ and negative $(\beta$ and $\lambda)$ effects of the selling price and product quality on supply chain performance. Conditions (10) and (11) also assure the non-negativity of solutions and the concavity of profit functions in all models. Applying $p^{F I^{*}}$ and $q^{F I^{*}}$ in (1) and (4) also obtains $D^{F I^{*}}$ and $\Pi_{R M S} F I^{*}$ (and $\Pi^{F I^{*}}$ ). The solutions of all cases are summarized in Tables 1 and 2, in Section 3.6. 


\subsection{Case $R M$ : integration of $R \& D$ and manufacturing processes}

R\&D or the manufacturer in Case RM integrates both R\&D and manufacturing processes, such as in the case of Sony Co. Alternatively, Case RM can also be regarded as a situation in which the manufacturer's action is coordinated under R\&D's monitoring and control, as in the case of the close relationship between Apple Inc. and Foxconn Technology Group; the opposite occurs in the relationship between Toyota Motor Co. and Denso Co., an automotive component R\&D and supplier. The player, by integrating two processes, plays the roles of both $R \& D$ and manufacturer (RM); while the individual seller's profit $\Pi_{S}{ }^{R M}=\Pi_{S}$ in (4), its profit $\Pi_{R M}{ }^{R M}$ is as follows:

$$
\Pi_{R M}{ }^{R M}=\Pi_{r}+\Pi_{m}=T_{s m}-c D-\lambda q^{2}=(w-c) D-\lambda q^{2},
$$

as $T_{s m}=w D$ in (5). The superscript $R M$ denotes Case RM.

In this decentralized system, players demonstrate the same opportunistic behavior as in practice, maximizing their own profit. The two-stage decision structure of Case RM can then be defined, based on the principal-agent paradigm:

$$
\begin{array}{ll}
\text { Maximize } & \Pi_{R M}{ }^{R M}(q, w) ; \\
\text { subject to } & \Pi_{S}^{R M}(p \mid q, w)>0 ; \\
& \text { Maximize } \Pi_{S}^{R M}(p \mid q, w) .
\end{array}
$$

The company conducting both $R \& D$ and manufacturing (RM) maximizes its own profit in (13), but needs to satisfy constraints (14) and (15). Inequality (14) represents the seller's rationality. The seller participates in the contract guaranteeing the positive profit. Constraint (15) is the seller's incentive compatibility constraint, in which the seller determines $p$, maximizing its own profit given the decisions of $q$ and $w$.

Through backward induction, we first obtain the response of the seller $p^{R M}$, given $q$ and $w$ from the FONC of (15), by partially differentiating $\Pi_{S}{ }^{R M}$ with respect to $p$ :

$$
p^{R M}(q, w)=\frac{\alpha+\beta w+\gamma q}{2 \beta} .
$$

After applying $p^{R M}$ to (13), and by simultaneously solving the FONCs of (13) from the partial differentiation with respect to $q$ and $w$, we obtain the optimal quality and contract term:

$$
\begin{aligned}
q^{R M^{*}} & =\frac{\gamma(\alpha-\beta c)}{8 \beta \lambda-\gamma^{2}}, \text { and } \\
w^{R M^{*}} & =\frac{4 \lambda(\alpha-\beta c)}{8 \beta \lambda-\gamma^{2}}+c .
\end{aligned}
$$

The solution of Case RM is summarized in Tables 1 and 2 in Section 3.6. 


\subsection{Case MS-R: integration of manufacturing and sales processes with $R \& D$ as the Stackelberg leader}

Case MS-R represents a supply chain in which the manufacturer directly sells the product by integrating the sales process (e.g., the direct sales of Dell, Inc.). This can also be regarded as a situation in which the manufacturer coordinates the seller's action. In Case MS-R, R\&D has bargaining power over the manufacturer, and thus determines the contract term. An example is Qualcomm, Inc., which owns a number of essential technology patents in the telecommunications industry. The following assumption is added to characterize the transaction between $R \& D$ and the manufacturer:

Assumption 4. When $R \& D$ has bargaining power over the manufacturer, $R \& D$ offers the technology transfer contract based on per unit royalty, while the manufacturer with bargaining power prefers the contract to be based on a fixed fee, independent of demand.

The two common payment modes for technology transfer in practice are per unit royalty and a fixed fee independent of the quantity produced. When patent licensing is related to quality innovation, Stamatopoulos and Tauman (2008), and Li and Wang (2010) have revealed that per unit royalty licensing is superior to fixed fee licensing from the external R\&D firm's perspective. Thus, we suppose that R\&D (that holds the power) offers a contract to the manufacturer based on per unit royalty in exchange for the transfer of its $R \& D$ results, with the expectation of continuous revenue over the product's life cycle. We assume that the one-time fixed fee contract is preferred when the manufacturer has bargaining power over R\&D, as is commonly observed in the technology acquisition of large manufacturing firms. Assumption 4 will be verified later, in Proposition 1.

The total transfer payment to R\&D in Case MS-R is defined as follows, where $r$ is the per unit royalty determined by $R \& D$ :

$$
T_{m r}^{M S-R}=r D
$$

The profit of the manufacturer who integrates the sales process (MS) $\Pi_{M S}^{M S-R}$ is defined as follows:

$$
\Pi_{M S}^{M S-R}=\Pi_{m}+\Pi_{s}=(p-c) D-T_{m r}=(p-c-r) D .
$$

R\&D's profit $\Pi_{R}{ }^{M S-R}=\Pi_{r}$, as defined in (2).

Thus, the problem of Case MS-R can be defined as follows:

$$
\begin{array}{ll}
\text { Maximize } & \Pi_{R}^{M S-R}(q, r) ; \\
\text { subject to } & \Pi_{M S}^{M S-R}(p \mid q, r)>0 ; \\
& \text { Maximize } \Pi_{M S}^{M S-R}(p \mid q, r) .
\end{array}
$$

Through an approach similar to Case RM, which involves a two-stage backward induction and is based on differential calculus, we can obtain the optimal selling price, product quality, and unit royalty of Case MS-R. The optimal solution is summarized in Tables 1 and 2 in Section 3.6. 


\subsection{Case MS-M: integration of manufacturing and sales processes with the manufacturer as the Stackelberg leader}

Case MS-M also considers the integration of manufacturing and sales processes, as in Case MS-R, but the manufacturer now has the bargaining power. Therefore, the manufacturer outsources $R \& D$, and offers the fixed-fee contract for R\&D output transfer, as described in Assumption 4. The fee is proportional to the resulting product quality $q$, and is independent of demand. Therefore, the fixed fee for technology transfer from the manufacturer to R\&D is defined in Case MS-M as:

$$
T_{m r} M S-M=f q
$$

where $f$ is the marginal fixed fee of the resulting quality. The manufacturer's profit $\Pi_{M S}{ }^{M S-M}$ is then defined as follows, while R\&D's profit $\Pi_{R}{ }^{M S-R}=\Pi_{r}$ is in given in (2):

$$
\Pi_{M S}^{M S-M}=\Pi_{m}+\Pi_{s}=(p-c) D-T_{m r}=(p-c) D-f q .
$$

The problem of Case MS-M can then be defined as follows:

$$
\begin{array}{ll}
\text { Maximize } & \Pi_{M S}{ }^{M S-M}(p, f) \\
\text { subject to } & \Pi_{R}^{M S-R}(q \mid p, f)>0 ; \\
& \text { Maximize } \Pi_{R}^{M S-R}(q \mid p, f) .
\end{array}
$$

The optimal solution is summarized in Tables 1 and 2 in Section 3.6.

\subsection{Case PD-R: pure decentralization with R\&D as the Stackelberg leader}

Case PD-R represents a supply chain in which all players maximize their own profits, while $R \& D$ is the focal company. All players in this purely decentralized system are responsible only for their basic processes, that is, $\Pi_{R}^{P D-R}=\Pi_{r}$ in (2), $\Pi_{M}^{P D-R}=\Pi_{m}$ in (3), and $\Pi_{S}{ }^{P D-R}=\Pi_{S}$ in (4).

$\mathrm{R} \& \mathrm{D}$ in Case PD-R offers a per unit royalty contract, as in Case MS-R; thus, $T_{m r} P D-R=$ $r D$ as in (19). Therefore, the three-stage decision structure of Case PD-R is defined as follows:

$$
\begin{array}{ll}
\text { Maximize } & \Pi_{R}{ }^{P D-R}(q, r) ; \\
\text { subject to } & \Pi_{M}(w \mid q, r)>0 ; \\
& \text { Maximize } \Pi_{M}{ }^{P D-R}(w \mid q, r) ; \\
& \text { subject to } \Pi_{S}^{P D-R}(p \mid q, r, w)>0 ; \\
& \text { Maximize } \Pi_{S}{ }^{P D-R}(p \mid q, r, w) .
\end{array}
$$

Each player's best response is obtained by following a solution approach similar to the former decentralization cases, but by conducting the backward induction twice. 


\subsection{Case PD-M: Pure decentralization with the manufacturer as the Stackelberg leader}

Case PD-M considers the same structure as Case PD-R, but the manufacturer has the bargaining power. Therefore, the manufacturer offers $R \& D$ a fixed fee contract for technology transfer, that is, $T_{m r} P D-M=f q$ as in (24), and controls the seller by offering a wholesale price contract. The two-stage optimization problem of Case PD-M with two agents, considering the dual role of the manufacturer as a control center, can be defined as:

$$
\begin{array}{ll}
\text { Maximize } & \Pi_{M}^{P D-M}(f, w) \\
\text { subject to } & \Pi_{R}{ }^{P D-M}(q \mid f, w)>0 \\
& \text { Maximize } \Pi_{R}{ }^{P D-M}(q \mid f, w) \\
& \Pi_{S}{ }^{P D-M}(p \mid f, w)>0 \\
& \text { Maximize } \Pi_{S}{ }^{P D-M}(p \mid f, w) .
\end{array}
$$

\begin{tabular}{|c|c|c|c|c|c|}
\hline \multirow{2}{*}{ Case } & \multicolumn{2}{|c|}{ Contract } & \multicolumn{2}{|c|}{ Decision } & \multirow{2}{*}{$\begin{array}{c}\begin{array}{c}\text { Consumer } \\
\text { response }\end{array} \\
D^{*}\end{array}$} \\
\hline & $r^{*}$ or $f^{*}$ & $w^{*}$ & $p^{*}$ & $q^{*}$ & \\
\hline \multirow[b]{2}{*}{ FI } & \multirow{2}{*}{ N/A } & \multirow{2}{*}{ N/A } & $2 \lambda(\alpha-\beta c)$ & $\gamma(\alpha-\beta c)$ & $2 \beta \lambda(\alpha-\beta c)$ \\
\hline & & & $4 \beta \lambda-\gamma^{2}$ & $\overline{4 \beta \lambda-\gamma^{2}}$ & $4 \beta \lambda-\gamma^{2}$ \\
\hline \multirow{2}{*}{$\mathrm{RM}$} & \multirow{2}{*}{ N/A } & $4 \lambda(\alpha-\beta c)$ & $6 \lambda(\alpha-\beta c)$ & $\underline{\gamma(\alpha-\beta c)}$ & $2 \beta \lambda(\alpha-\beta c)$ \\
\hline & & $8 \beta \lambda-\gamma^{2}$ & $8 \beta \lambda-\gamma^{2}$ & $8 \beta \lambda-\gamma^{2}$ & $8 \beta \lambda-\gamma^{2}$ \\
\hline \multirow{2}{*}{ MS-R } & $4 \lambda(\alpha-\beta c)$ & \multirow{2}{*}{ N/A } & $6 \lambda(\alpha-\beta c)$ & $\gamma(\alpha-\beta c)$ & $2 \beta \lambda(\alpha-\beta c)$ \\
\hline & $8 \beta \lambda-\gamma^{2}$ & & $8 \beta \lambda-\gamma^{2}$ & $8 \beta \lambda-\gamma^{2}$ & $8 \beta \lambda-\gamma^{2}$ \\
\hline \multirow{2}{*}{ MS-M } & $2 \gamma \lambda(\alpha-\beta c)$ & \multirow{2}{*}{ N/A } & $4 \lambda(\alpha-\beta c)$ & $\gamma(\alpha-\beta c)$ & $4 \beta \lambda(\alpha-\beta c)$ \\
\hline & $\overline{8 \beta \lambda-\gamma^{2}}$ & & $8 \beta \lambda \lambda-\gamma^{2}$ & $\overline{8 \beta \lambda-\gamma^{2}}$ & $8 \beta \lambda-\gamma^{2}$ \\
\hline \multirow{2}{*}{ PD-R } & $8 \lambda(\alpha-\beta c)$ & $12 \lambda(\alpha-\beta c)$ & $14 \lambda(\alpha-\beta c)$ & $\gamma(\alpha-\beta c)$ & $2 \beta \lambda(\alpha-\beta c)$ \\
\hline & $\overline{16 \beta \lambda-\gamma^{2}}$ & $16 \beta \lambda-\gamma^{2}$ & $\overline{16 \beta \lambda-\gamma^{2}}$ & $\overline{16 \beta \lambda-\gamma^{2}}$ & $16 \beta \lambda-\gamma^{2}$ \\
\hline \multirow{2}{*}{ PD-M } & $2 \gamma \lambda(\alpha-\beta c)$ & $8 \lambda(\alpha-\beta c)$ & $12 \lambda(\alpha-\beta c)$ & $\gamma(\alpha-\beta c)$ & $4 \beta \lambda(\alpha-\beta c)$ \\
\hline & $16 \beta \lambda-\gamma^{2}$ & $\overline{16 \beta \lambda-\gamma^{2}}$ & $16 \beta \lambda-\gamma^{2}$ & $\overline{16 \beta \lambda-\gamma^{2}}$ & $16 \beta \lambda-\gamma^{2}$ \\
\hline
\end{tabular}

The solutions of all models are summarized in Tables 1 and 2.

Table 1. Solution summary: variables 
Table 2. Solution summary: profits

\begin{tabular}{|c|c|c|c|c|}
\hline Case & $\begin{array}{l}\mathrm{R} \& \mathrm{D} \\
\Pi_{R}^{*}\end{array}$ & $\begin{array}{c}\text { Manufacturer } \\
\Pi_{M}{ }^{*} \\
\end{array}$ & $\begin{array}{c}\text { Seller } \\
\Pi_{S}{ }^{*}\end{array}$ & $\begin{array}{c}\text { Supply chain } \\
\Pi^{*}\end{array}$ \\
\hline FI & & ${ }_{M S}^{I^{*}}=\frac{\lambda(\alpha-\beta c)^{2}}{4 \beta \lambda-\gamma^{2}}$ & & $\frac{\lambda(\alpha-\beta c)^{2}}{4 \beta \lambda-\gamma^{2}}$ \\
\hline $\mathrm{RM}$ & $\Pi_{R M}^{R M^{*}}=$ & $\frac{-\beta c)^{2}}{-\gamma^{2}}$ & $\frac{4 \beta \lambda^{2}(\alpha-\beta c)^{2}}{\left(8 \beta \lambda-\gamma^{2}\right)^{2}}$ & $\frac{\lambda\left(12 \beta \lambda-\gamma^{2}\right)(\alpha-\beta c)^{2}}{\left(8 \beta \lambda-\gamma^{2}\right)^{2}}$ \\
\hline MS-R & $\frac{\lambda(\alpha-\beta c)^{2}}{8 \beta \lambda-\gamma^{2}}$ & $\Pi_{M S}^{M S-R^{*}}=$ & $\frac{\lambda^{2}(\alpha-\beta c)^{2}}{\left.\beta \lambda-\gamma^{2}\right)^{2}}$ & $\frac{\lambda\left(12 \beta \lambda-\gamma^{2}\right)(\alpha-\beta c)^{2}}{\left(8 \beta \lambda-\gamma^{2}\right)^{2}}$ \\
\hline MS-M & $\frac{\lambda \gamma^{2}(\alpha-\beta c)^{2}}{\left(8 \beta \lambda-\gamma^{2}\right)^{2}}$ & $\Pi_{M S}^{M S-M^{*}}=$ & $\frac{\lambda(\alpha-\beta c)^{2}}{8 \beta \lambda-\gamma^{2}}$ & $\frac{\lambda\left(16 \beta \lambda-\gamma^{2}\right)(\alpha-\beta c)^{2}}{\left(8 \beta \lambda-\gamma^{2}\right)^{2}}$ \\
\hline PD-R & $\frac{\lambda(\alpha-\beta c)^{2}}{16 \beta \lambda-\gamma^{2}}$ & $\frac{8 \beta \lambda^{2}(\alpha-\beta c)^{2}}{\left(16 \beta \lambda-\gamma^{2}\right)^{2}}$ & $\frac{4 \beta \lambda^{2}(\alpha-\beta c)^{2}}{\left(16 \beta \lambda-\gamma^{2}\right)^{2}}$ & $\frac{\lambda\left(28 \beta \lambda-\gamma^{2}\right)(\alpha-\beta c)^{2}}{\left(16 \beta \lambda-\gamma^{2}\right)^{2}}$ \\
\hline PD-M & $\frac{\gamma^{2} \lambda(\alpha-\beta c)^{2}}{\left(16 \beta \lambda-\gamma^{2}\right)^{2}}$ & $\frac{2 \lambda(\alpha-\beta c)^{2}}{16 \beta \lambda-\gamma^{2}}$ & $\frac{16 \beta \lambda^{2}(\alpha-\beta c)^{2}}{\left(16 \beta \lambda-\gamma^{2}\right)^{2}}$ & $\frac{\lambda\left(48 \beta \lambda-\gamma^{2}\right)(\alpha-\beta c)^{2}}{\left(16 \beta \lambda-\gamma^{2}\right)^{2}}$ \\
\hline
\end{tabular}

\section{Comparison of supply chain structures}

This section reveals the unique characteristics of the supply chain models. First, Assumption 4 regarding the payment mode selection for technology transfer is verified.

Proposition 1. If the fixed fee contract is adopted in Cases MS-R and PD-R, this does not guarantee the concavity of $\Pi_{R}$, while the per unit royalty contract also does not guarantee the concavity of $\Pi_{M}$ in Cases MS-M and PD-M.

Proof of Proposition 1. In Cases MS-R, the Hessian determinants of $\Pi_{R}$ are $\left|\mathrm{H}_{1}\right|=$ $\left(\partial^{2} \Pi_{R} / \partial q^{2}\right)=-2 \lambda<0$ and $\left|\mathrm{H}_{2}\right|=\left(\partial^{2} \Pi_{R} / \partial q^{2}\right)\left(\partial^{2} \Pi_{R} / \partial f^{2}\right)-\left(\partial^{2} \Pi_{R} / \partial q \partial f\right)^{2}=-1<0$, if we apply $T_{m r}=f q$ in (24). They do not satisfy the negative-definite property of the secondorder sufficient condition, and hence profit maximization is not guaranteed. This is similar to Case PD-R, which also shows $\left|\mathrm{H}_{1}\right|<0$ and $\left|\mathrm{H}_{2}\right|<0$ if we apply $T_{m r}=f q$. In Cases MS-M and PD-M, the concavity of $\Pi_{M}$ is also not guaranteed if we apply $T_{m r}=$ $r D$. Therefore, Proposition 1 holds.

When R\&D has power over the manufacturer, it can maximize its own profit by offering a per unit royalty contract, which cannot be done with a fixed fee contract. This explains why the per unit royalty scheme is a common licensing contract of patent technology in practice, when a patent-holding company such as Qualcomm has stronger bargaining power. Offering a per unit royalty contract to external $R \& D$ is not a reasonable choice for the manufacturer, while a fixed fee contract guarantees the manufacturer's profit maximization. As we can observe in practice, manufacturing companies with power, such as Samsung Electronics, are not willing to pay per unit royalty for the lifecycle of a product to an R\&D firm. Instead, they prefer to acquire the technology at a one-time 
fixed payment, or sometimes even try to merge with a firm owning the patent technology, such as Harman International Industries with audio technology. Next, we characterize the interaction among players in the supply chain models.

Proposition 2. The following properties are obtained by investigating the transfer payments:

$$
\begin{aligned}
& \text { (1) } r^{M S-R^{*}}>r^{P D-R^{*}} \text { and } f^{M S-M^{*}}>f^{P D-M^{*}} \text {, and } \\
& \text { (2) } w^{P D-R^{*}}>w^{R M^{*}}>w^{P D-M^{*}} \text {. }
\end{aligned}
$$

Proof of Propositions 2-4. The results of Propositions 2-4 are obtained by direct comparison of the optimal solutions summarized in Tables 1 and 2 . For example, $r^{M S-R^{*}}$ $r^{P D-R^{*}}=4 \gamma^{2} \lambda(\alpha-\beta c) /\left[\left(8 \beta \lambda-\gamma^{2}\right)\left(16 \beta \lambda-\gamma^{2}\right)\right]$, and it yields $r^{M S-R^{*}}>r^{P D-R^{*}}$, based on $\alpha>\beta c$ in Condition (10) and $4 \beta \lambda-\gamma^{2}>0$ in Condition (11). This establishes the result of Proposition 2(1). Following similar approaches by direct comparison, we obtain the results of Propositions 2-4.

The transfer payment for the R\&D result from the manufacturer to $R \& D$ is always greater in the integrated supply chain structure (Case MS-R or MS-M) than in the decentralized system (Case PD-R or PD-M), as noted in Proposition 2(1). This relationship holds, regardless of a payment type of royalty per unit sold (Cases MS-R and PD-R) or fixed fee (Cases MS-M and PD-M). This implies that manufacturing firms like Tesla can take advantage of a better understanding of the consumer market; it can integrate sales and service to target a premium market with a higher quality product through better facilitation of investment in quality by an outside R\&D firm such as Panasonic with battery technologies. The wholesale price $w$ is also determined differently, depending not only on the supply chain structure, but also on the power dynamics between players, as in Proposition 2(2). $w$ is determined as the largest in Case PD-R, while it is the smallest in Case PD-M. The highest $w$ in the R\&D-led decentralized system (Case PD$\mathrm{R}$ ) is due to the three-stage decision structure and innate triple marginalization issues, as observed in the problem structure in Section 3.5. On the other hand, the lowest $w$ in the manufacturer-led decentralized system (Case PD-M) is because the manufacturer can mitigate marginalization issues by centralized control, directly controlling opportunistic behaviors of both R\&D and the seller, as seen in Section 3.6.

These different interactions between players then induce different R\&D performance and selling price decisions.

Proposition 3. The following properties are obtained by comparing product quality and selling prices:

$$
\begin{aligned}
& \text { (1) } q^{F I^{*}}>q^{R M^{*}}=q^{M S-R^{*}}=q^{M S-M^{*}}>q^{P D-R^{*}}=q^{P D-M^{*}} \text {, and } \\
& \text { (2) } p^{P D-R^{*}}>p^{R M^{*}}=p^{M S-R^{*}}>p^{P D-M^{*}}>p^{F I^{*}}>p^{M S-M^{*}} \text {. }
\end{aligned}
$$

Figure 2 graphically illustrates the results of Proposition 3. In Proposition 3(1) and as shown in Figure 2, product quality $q$, representing R\&D performance, is a function of the supply chain structure. The resulting $q$ is clearly differentiated by the level of process integration. Specifically, the highest value of $q$ is expected in the fully coordinated 
system (Case FI), while the purely decentralized supply chain always yields the lowest value of $q$ (Cases PD-R and PD-M). This illustrates that the level of process integration is a critically affects product quality. If a company prioritizes the premium market by targeting a consumer segment that prefers a high-quality product, it is advantageous to maintain an integrated system that closely coordinates overall supply chain processes and decisions, not only in R\&D and manufacturing, but also in sales. Tesla announced in 2014 that it decided to internalize the R\&D and production of battery cells - of which many parts were formerly outsourced to Panasonic - by building its Gigafactory in Nevada, U.S. in cooperation with Panasonic. It aims to produce electric vehicles in sufficient numbers, and to accelerate the R\&D of high-quality batteries and innovative manufacturing through this cooperation. On the other hand, it is also interesting to observe that the results are completely independent of the supply chain's power dynamics. Note that R\&D's bargaining power does not affect R\&D performance, that is, $q^{M S-R^{*}}=$ $q^{M S-M^{*}}$, and $q^{P D-R^{*}}=q^{P D-M^{*}}$.

Further, note that the selling price $p$ in Proposition 3(2) and Figure 2 is a function of the supply chain's power dynamics. $p$ is higher when $R \& D$ controls the supply chain (Cases MS-R and PD-R), which is also noted in the solid-line area of Figure 2. This is due to multiple marginalization issues in the R\&D-led supply chain system, as explained in Proposition 2(2). However, $p$ can decrease if the manufacturer has the bargaining power (Cases MS-M and PD-M), as noted in the dotted-line area of Figure 2. This is because the manufacturer is located in the middle of the supply chain between $R \& D$ and the seller, and can directly control the overall supply chain's actions, as displayed in Figures 1 (d) and (f). Additionally, the level of coordination does not coincide with a lower selling price; we observe that the fully coordinated system without marginalization issues can yield a higher selling price than in the decentralized case, that is, $p^{F I^{*}}>$ $p^{M S-M^{*}}$. This results from the higher product quality in Case FI. However, higher quality does not always translate into a higher selling price.

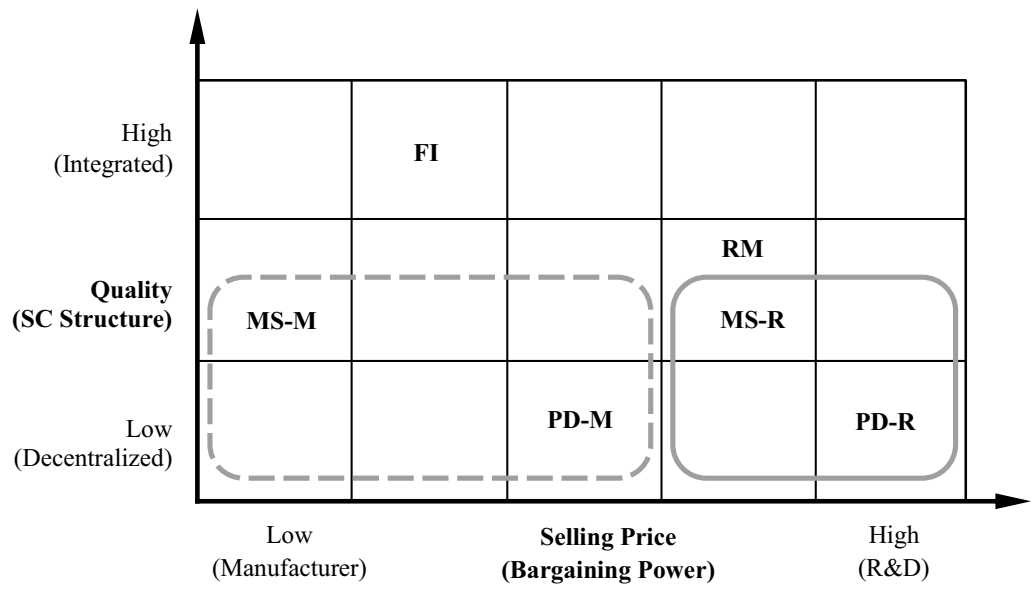

Fig. 2. Comparison of result of product quality and selling price in Proposition 3 (solid line: R\&D controls; dotted line: manufacturer controls) 
Overall, supply chain structure and power dynamics determine a product's quality and selling price. Each supply chain model's unique decision structure differently influences market and profit performance.

Proposition 4. The following properties are found by comparing demand requests and the supply chain's overall profits:

(1) $D^{F I^{*}}>D^{M S-M^{*}}>D^{R M^{*}}=D^{M S-R^{*}}>D^{P D-M^{*}}>D^{P D-R^{*}}$, and

(2) $\Pi^{F I^{*}}>\Pi^{M S-M^{*}}>\Pi^{R M^{*}}=\Pi^{M S-R^{*}}>\Pi^{P D-M^{*}}>\Pi^{P D-R^{*}}$.

Proposition 4 indicates that the market and profit performances are functions of both the supply chain structure and power dynamics, while a comparison of results of the two performance measures appear identical. Specifically, the supply chain structure or the level of process integration is the main determinant of performance, that is, both market and profit performances of a fully integrated system $\{$ Case FI $\}>$ partially integrated systems $\{$ MS-M, RM, MS-R\} > a purely decentralized system \{PD-M, PD-R\}. The power dynamics again differentiate the performances, while the manufacturer-led system yields a superior result to the R\&D-led system, that is, the performances of MS-M $>$ MS-R = RM, while PD-M > PD-R. This is due to the ability of the manufacturer located in the middle of a supply chain - to mitigate inefficiency problems resulting from marginalization issues through centralized control.

$\mathrm{R} \& \mathrm{D}$ is located at the upstream end of the supply chain, making it difficult to similarly play the role of a control center, as noted in the decision structure in Section 3.5. Therefore, the multiple marginalization problems of an R\&D-led system can make the overall system inefficient. These would worsen, as the structure of a supply chain is more complicated in practice. Therefore, in practice, it is critical for the specialized R\&D firm to systematically understand the supply chain's overall processes and interactions to find a way to control other players' actions. Focusing on marketing as well as R\&D - two core processes at the supply chain's downstream and upstream ends - can be one solution. This will help firms to effectively understand and control the entire supply chain, as in the cases of Apple, Inc. and Nike, Inc.

It is also worth observing that Cases RM and MS-R perform equally in terms of quality, price, demand, and supply chain profit, in spite of their different value chain structures. This indicates that $R \& D$ can choose the supply chain structure while maintaining the same performance.

\section{Conclusions}

In recent business environments, a common practice is supply chain-based business management, where functions decentralized to specialized partners. The R\&D function is no exception as increasing complexity of technologies require a firm to be more dependent upon its partners' complementary technological capabilities. When a firm forms a supply chain with its partners, it becomes of great importance in business practices to understand the effect of supply chain structure and players' power dynamics on a supply 
chain's R\&D and market performances. To the best of our knowledge, this study is the first to investigate these issues. Thus, this study contributes to the literature by bridging the gap between practice and academia, while providing valuable practical insights to the firms forming supply chains.

In this study, R\&D performance is investigated in a multi-tier supply chain context, consisting of a specialized R\&D firm, a manufacturer, and a seller. Six different types of supply chain models, differing in process integration and power dynamics, are introduced. Then, the comparison of their R\&D, market, and profit performance provides not only important implications for R\&D practice but also practical guidelines for practicing managers in a decentralized supply chain system. These can be summarized as follows.

First, a different payment mode for technology transfer must be selected, relative to the power dynamics in a supply chain. When $R \& D$ has bargaining power, it can maximize its own profit by a per unit royalty contract, while the fixed fee contract guarantees the manufacturer's profit maximization. Second, interactions among players are affected by both supply chain structure and power dynamics. The transfer payment for R\&D output is greater in the integrated than in the decentralized structure. The wholesale price is higher when $R \& D$ has bargaining power, due to innate multiple marginalization issues. Third, product quality, or R\&D performance is a function of the supply chain structure, higher in more integrated supply chains. Therefore, it is very important to design the structure of a supply chain based on the careful consideration of the market we target. A company targeting a premium market segment must closely coordinate overall supply chain decisions in an integrated system to yield a high-quality product. Further, note that power dynamics do not affect R\&D performance. Fourth, the selling price is a function of power dynamics in a supply chain. The R\&D-led supply chain yields a higher selling price, owing to marginalization issues; this can decrease if the manufacturer has the bargaining power, due to its centralized control over the supply chain. Fifth, the supply chain structure is the main determinant of market and profit performances, thereafter power dynamics differentiate the performances once again. The integrated, manufacturer-led system guarantees superior market and profit performance. The manufacturer's role must be revisited; it can mitigate marginalization issues by directly coordinating or controlling the overall supply chain's actions, based on its location between R\&D and the seller. Sixth, it is important for the R\&D firm to resolve multiple marginalization issues by systematically understanding the entire system. One solution would involve commanding both $\mathrm{R} \& \mathrm{D}$ and marketing, two core processes at the upstream and downstream ends.

While this study has important implications for research and practice, its limiting assumptions need to be relaxed in a future study. First, we have assumed that either R\&D or the manufacturer has the bargaining power in the supply chain. However, many supply chains are led by the seller, who is better positioned to understand consumer behavior. Therefore, it will be interesting to compare the seller-led model. Second, only the wholesale price contract was considered for the product supply. However, there are various types of contracts, which will differently affect overall performance. Third, we have not considered two possible supply chain cases from our study, involving the in- 
tegration of $R \& D$ and sales processes with either $R \& D$ or the manufacturer as a leader. Its inclusion may require us to additionally consider the production performance, such as the conformance of quality to design specification, while the present study focuses on the R\&D and market performances. A study that investigates these two cases, based on the present study's results, will enhance the implications for supply chain practice, and for effectively understanding supply chains such as Apple and Li \& Fung. Fourth, we have adopted a quadratic function form for R\&D investment, which is very common in many previous studies of quality management. However, if a further study adopts a general convex function or other types of function forms, it would provide more generalized implications for supply chain practice. Incorporating the above issues in a future study will allow the development of practical implications that better correspond to the current supply chain environment.

\section{References}

Banker, R. D.; Khosla, I.; Sinha, K. K. 1998. Quality and competition, Management Science 44(9): 1179-1192. https://doi.org/10.1287/mnsc.44.9.1179

Cachon, G. P.; Lariviere, M. A. 2005. Supply chain coordination with revenue-sharing contracts: strengths and limitations, Management Science 51(1): 30-44.

https://doi.org/10.1287/mnsc.1040.0215

Carr, S. M.; Karmarkar, U. S. 2005. Competition in multiechelon assembly supply chains, Management Science 51(1): 45-59. https://doi.org/10.1287/mnsc.1040.0216

Corbett, C. J.; Karmarkar, U. S. 2001. Competition and structure in serial supply chains with deterministic demand, Management Science 47(7): 966-978.

https://doi.org/10.1287/mnsc.47.7.966.9799

Ding, D.; Chen, J. 2008. Coordinating a three level supply chain with flexible return policies, Omega 36(5): 865-876. https://doi.org/10.1016/j.omega.2006.04.004

Han, S. Y.; Bae, S. J. 2014. Internalization of R\&D outsourcing: an empirical study, International Journal of Production Economics 150: 58-73. https://doi.org/10.1016/j.ijpe.2013.12.001

Kamien, M. I.; Oren, S. S.; Tauman, Y. 1992. Optimal licensing of cost-reducing innovation, Journal of Mathematical Economics 21(5): 483-508. https://doi.org/10.1016/0304-4068(92)90036-7

Karmarkar, U. S.; Pitbladdo, R. C. 1997. Quality, class, and competition, Management Science 43(1): 27-39. https://doi.org/10.1287/mnsc.43.1.27

Kishimoto, S.; Watanabe, N. 2017. The kernel of a patent licensing game: the optimal number of licensees, Mathematical Social Sciences 86: 37-50.

https://doi.org/10.1016/j.mathsocsci.2017.01.001

Kishimoto, S.; Watanabe, N.; Muto, S. 2011. Bargaining outcomes in patent licensing: asymptotic results in a general cournot market, Mathematical Social Sciences 61(2): 114-123.

https://doi.org/10.1016/j.mathsocsci.2010.12.001

Li, C.; Wang, J. 2010. Licensing a vertical product innovation, Economic Record 86(275): $517-$ 527. https://doi.org/10.1111/j.1475-4932.2010.00630.x

Lu, Q.; Meng, F.; Goh, M. 2014. Choice of supply chain governance: self-managing or outsourcing?, International Journal of Production Economics 154(1): 32-38.

https://doi.org/10.1016/j.ijpe.2014.03.022

Majumder, P.; Srinivasan, A. 2008. Leadership and competition in network supply chains, Management Science 54(6): 1189-1204. https://doi.org/10.1287/mnsc.1070.0752 
PhoneArena. 2015. Qualcomm hit hard by the Exynos chipset in Galaxy S6, might spin off the Snapdragon division [online], [cited 10 September 2016]. PhoneArena.com. Available from Internet: http://www.phonearena.com/news/Qualcomm-hit-hard-by-the-Exynos-chipset-in-Galaxy-S6might-spin-off-the-Snapdragon-division_id68359

Raz, G.; Ovchinnikov, A. 2015. Coordinating pricing and supply of public interest goods using government rebates and subsidies. IEEE Transactions on Engineering Management 62(1): 65-79. https://doi.org/10.1109/TEM.2014.2380999

Savaskan, R. C.; van Wassenhove, L. N. 2006. Reverse channel design: the case of competing retailers, Management Science 52(1): 1-14. https://doi.org/10.1287/mnsc.1050.0454

Sen, D. 2005. Fee versus royalty reconsidered, Games and Economic Behavior 53(1): 141-147. https://doi.org/10.1016/j.geb.2004.09.005

Shi, L.; Ólafsson, S.; Chen, Q. 2001. An optimization framework for product design, Management Science 47(12): 1681-1692. https://doi.org/10.1287/mnsc.47.12.1681.10243

Shi, W.; Min, K. J. 2013. A study of product weight and collection rate in closed-loop supply chains with recycling, IEEE Transactions on Engineering Management 60(2): 409-423.

https://doi.org/10.1109/TEM.2012.2214222

Spengler, J. J. 1950. Vertical integration and antitrust policy, Journal of Political Economy 58(4): 347-352. https://doi.org/10.1086/256964

Stamatopoulos, G.; Tauman, Y. 2008. Licensing of a quality-improving innovation, Mathematical Social Sciences 56(3): 410-438. https://doi.org/10.1016/j.mathsocsci.2008.06.006

Tian, X. 2016. Licensing a quality-enhancing innovation to an upstream firm, Economic Modelling 53: 509-514. https://doi.org/10.1016/j.econmod.2015.11.001

Wang, J.; Shin, H. 2015. The impact of contracts and competition on upstream innovation in a supply chain, Production and Operations Management 24(1): 134-146.

https://doi.org/10.1111/poms.12218

Wang, X. H. 2002. Fee versus royalty licensing in a differentiated Cournot duopoly, Journal of Economics and Business 54(2): 253-266. https://doi.org/10.1016/S0148-6195(01)00065-0

Yoo, S. H.; Kim, B. C. 2016. Joint pricing of new and refurbished items: a comparison of closedloop supply chain models, International Journal of Production Economics 182: 132-143. https://doi.org/10.1016/j.ijpe.2016.07.017

Yoo, S. H.; Kim, D.; Park, M-S. 2015. Pricing and return policy under various supply contracts in a closed-loop supply chain, International Journal of Production Research 53(1): 106-126. https://doi.org/10.1080/00207543.2014.932927

Zhao, Y.; Wang, S.; Cheng, T. E.; Yang, X.; Huang, Z. 2010. Coordination of supply chains by option contracts: a cooperative game theory approach, European Journal of Operational Research 207(2): 668-675. https://doi.org/10.1016/j.ejor.2010.05.017

Seung Ho YOO is an Associate Professor at Department of Business Administration, Sunmoon University in Korea. His research interest mainly lies in investigating supply chain issues, especially how to facilitate collaboration and improve overall performance by improving relationship structures among players.

Yong Won SEO is an Associate Professor at Department of Business Administration, Chung-Ang University in Seoul, Korea. He had earned his B.S., M.S., and PhD degrees at the Seoul National University in Korea. His research interest mainly lies in the operations management and supply chain management areas, especially in decision coordination, behavioral operations, and risk management models in supply chain operations. 